\title{
Können/müssen/sollten die Ergebnisse fachlich sinnvoller medizinischer Qualitätsindikatoren dem Patienten vermittelt werden - wenn ja, wie?
}

Seit dem Berichtsjahr 2004 liegen der Öffentlichkeit Berichte der 2000 Krankenhäuser in Deutschland über deren Qualität im Vergleich zu anderen Krankenhäusern vor. Der alle 2 Jahre vorzulegende Bericht verwendet teilweise Indikatoren der gesetzlichen Qualitätssicherung. Neben gedruckten Schriftstücken und solchen in druckähnlichen elektronischen Formaten werden die Zahlenwerke auch durch eine Reihe von internetbasierten Datenbankabfrage-Websites aufbereitet.

An der ersten Erstellung der Berichte waren weder Patienten noch Patientenvertretungen beteiligt. Auch im aktuellen Verfahren beschränkt sich deren Beteiligung auf eine stimmrechtlose Mitberatung. Dass in diesem Jahr erstmals von der verantwortlichen Selbstverwaltung die Evaluation in Form einer Nutzerbefragung beschlossen wurde, mag auf diesem Hintergrund überraschen. Ebenso neu ist im Zusammenhang mit den Berichten die Herausgabe eines werbenden Faltblatts und eines Posters durch den Herausgeber „Gemeinsamer Bundesausschuss in Siegburg“. Damit sollen speziell künftige Patientinnen und Patienten durch einweisende Ärzte angesprochen und als Nutzer des Berichts gewonnen werden.

Die Berichte verwenden in hohem Maß die medizin-übliche Spezialsprache eines akademischen Standes, die Auswahl der vergleichbaren Indikatoren richtete sich nach dem Interesse der an der Auswahl beteiligten Interessenvertretungen von Krankenkassen und Krankenhäusern.

Befragungen von Patientinnen und Patienten $[1,2]$ zu diesen Berichten sowie vergleichbaren Werken aus dem Bereich der Rehabilitation ergaben einerseits ein großes öffentliches Interesse an Informationen über die Qualität einer künftig zur Behandlung vorgesehenen Einrichtung, andererseits ein Überangebot aus Sicht von solchen Nutzerinnen und Nutzern unnötiger Informationen. Das Fehlen von Bewertungen aus Sicht von ehemaligen Patientinnen und Patienten wurde insgesamt als Strukturfehler der Berichte angesehen.

Wenn bei der Beurteilung berücksichtigt wird, dass es bis heute kein Verfahren zum Feststellen von unrichtigen Angaben gibt, dies vielmehr der Aufmerksamkeit der jeweiligen Konkurrenten im Wettbewerb und deren Zugang zu anderen Medien überlassen bleibt, ist der Wert bisheriger Werke eingeschränkt.
Künftig sollten neben einem beschränkten Satz an Indikatoren, deren Auswahl transparent dargestellt werden muss, die Ergebnisse von Nutzerbefragungen dargestellt werden. Beiderlei Ergebnisse sind unabhängig von den Anbieterinteressen zu ermitteln und darzustellen. Datenbanknutzungen im Internet werden eine größere Rolle spielen wenn es um Vergleiche geht, entsprechend wird die Bedeutung der aufwändigen Druckstücke zurückgehen.

Autorenerklärung: Der Autor erklärt, dass keine relevanten finanziellen Verbindungen in Bezug auf dieses Manuskript bestehen.

Literatur

1 Schwartze D, Geraedts M. Eignung von Qualitätsindikatoren und grafischen Qualitätsvergleichen zur informierten Krankenhauswahl durch Patienten Das Gesundheitswesen 2006; 68: 489

2 Seidel G, Haase I, Walle E, Dierks ML. Ein Qualitätsbericht Rehabilitation aus der Sicht von Patienten und Verbrauchern [Poster]. 18. wissenschaftliches Kolloquium: Innovation in der Rehabilitation Kommunikation und Vernetzung Münster, 09.03.2009 - 11.03.2009
W.-D. Trenner

Qualitätsmanagement

Schlüsselwörter

Qualitätsindikatoren

Qualitätsbericht

Keywords

$\checkmark$ quality indicators

quality report
Institut

Fördergemeinschaft für Taubblinde e.V., Berlin

Bibliografie

DOI 10.1055/s-0029-1242683

Dtsch Med Wochenschr 2009; 134: S329 - (c) Georg Thieme Verlag KG Stuttgart - New York . ISSN 0012-0472

Korrespondenz Wolf-Dietrich Trenner Fördergemeinschaft für Taubblinde e. V. Katteweg 15a 14129 Berlin Tel. 030/8049-7461 eMail verein@taubblinde.de 